\title{
Preliminary evidence of dynamic muscular synergies in human grasping
}

\author{
Claudio Castellini and Patrick van der Smagt \\ Institute of Robotics and Mechatronics \\ DLR / German Aerospace Research Center \\ Oberpfaffenhofen, Germany \\ e-mail claudio.castellini@dlr.de, smagt@dlr.de
}

\begin{abstract}
Motor synergies have been investigated since the 1980s as a simplifying paradigm of motor control by the nervous system. In particular, it is believed that they allow control of the highly redundant kinematic chain of the human hand by the central nervous system. Whereas so far the focus has been on kinematic synergies, that is common patterns in the motion of the hand and fingers, we hereby also investigate their dynamic aspect, evaluated through surface electromyography. We especially show that dynamic motor synergies exist, i.e., that muscles are activated synergistically; and that these synergies are largely comparable to one another across human subjects, even though surface electromyography is usually disturbed by muscle crosstalk, sweating, anatomical differences and inaccurate electrode positioning. If confirmed, these results would have applications, e.g., in control of advanced robotic hands.
\end{abstract}

\section{INTRODUCTION}

The human hand has a very complex biomechanical structure, and a complicated neural architecture to control it. In order to replicate the functionality of the human hand in robotic systems, we need to understand, model, and finally copy the control strategies that underlie the coordination of digit movement and forces as necessary for a large number of tasks, including power grasps as well as fine manipulation. In the analysis of the biomechanical and behavioural aspects of the hand, one of the most striking aspects is the high redundancy of its structure, having many more degrees of freedom than actually necessary to do what it must.

In order to cope with this apparent redundancy, the aspect of synergies has been used to describe functional dependencies among degrees of freedom. Bernstein [1] defines the level responsible for coordinating large muscle groups and different movement patterns as the level of muscular-articular links or synergies. Thus the state space of the system can be reduced to a few dimensions, in order to "simplify the control problem at hand". A similar finding is the well-known combination of motor primitives in frogs by Mussa-Ivaldi et al. [2].

A number of recent experimental studies confirm these theories for the human hand. As shown by Santello et al. [3], [4], the simultaneous motion of the fingers is characterized by coordination and covariation patterns that reduce the number of independent degrees of freedom to be controlled. Although some constraints on the musculotendon system, as well as on the peripheral and central neural system, can be identified, a clear relationship between the finger kinematic constraints and the underlying muscular activity remains to be analysed.

The source of such kinematic synergies in the human hand remains a matter of debate. Indeed, the biomechanical structure of the hand, in which tendons activate multiple digits at the same time, while the related muscles share common bases, is one source for the synergies. But also the spinal coactivation circuitry, mapped only to a small extent for the human hand, coactivates muscles and thus defines synergies. At the highest level, cortical organisation is suspected to play a dominant but variable role in these.

Muscular activity measured via surface electromyography (sEMG from now on) relates nearly linearly to the force exerted by the muscles [5]. However, there is no such relationship between the muscle activity and the finger position. Therefore, a clear relationship between the finger position synergies as found in [3] and muscle synergies is not at all obvious. That means: the existence of kinematic motor synergies does not necessarily imply the existence of dynamic ones, although it is quite likely so. In this paper we show that such dynamic muscle synergies, that is, synergistic muscle activations, can be clearly identified while grasping 5 objects in 5 accordingly different ways; moreover, we show that there is considerable statistical overlapping among dynamic synergies found across 5 human subjects. In other words, all subjects seem to enact a certain grasp by activating the same muscles (or groups of motor units) all over.

Muscular activity is gathered using sEMG via 10 commercial prosthetic electrodes placed on the forearm of each of the subjects, with no anatomically precise positioning. The fact that common human dynamic synergies can be identified although sEMG is well-known to be disturbed by a number of factors (e.g., muscle cross-talk, sweating, anatomical differences, inaccurate electrode positioning) makes this result rather interesting. If confirmed on a larger data set, common dynamic synergies might be fruitfully employed in bioinspired robotics, especially in the control of highly dexterous robotic hands.

After describing our experimental setup in Sec. II, the results of our measurements are presented in Sec. III. Here we show two components of the synergies: $(a)$ the PCA-computed synergies between the objects, and $(b)$ the (linear!) separability 
of the grasps, when only 3 pincipal components of the EMG data are used, among all subjects.

\section{EXPERIMENT DESCRIPTION}

\section{A. Data gathering}

1) Hand motion: An 18-sensor right-handed Cyberglove (Cyberglove Systems, www.cyberglovesystems.com, see also Figure 1, left panel) is used to gather the finger positions. The Cyberglove is a light fabric, rather elastic glove, onto which 18 strain gauges are sewn; the sewing sheaths are chosen carefully by the manufacturer, so that the gauges exhibit a resistance which is proportionally related to the angles between pairs of hand joints of interest. The device can then return 18 8-bit values, proportional to these angles, for an average resolution of less than one degree, depending on the size of the subject's hand, a careful wearing of the glove and the rotation range of the considered joint. (For practical reasons the subject must wear a cotton glove below the Cyberglove; an initial round of data gathering revealed that this would not limit the precision of the device.) We hereby consider all sensor values of the glove, that is, 18 8-bit values. Figure 1, center panel shows the placement of the sensors on the 22 -sensors glove, which has 4 additional sensors at the distal phalanxes.

2) EMG and pressure: Muscular activity is gathered using OttoBock MyoBock 13E200 surface EMG electrodes (www. ottobock.com). The electrodes already provide an amplified, bandpass-filtered and rectified signal, eliminating the need of further processing onboard the card and/or the computer (their usefulness was already demonstrated at least in [5], [6]). They are connected to a DAQ card sampling the EMG signals at $100 \mathrm{~Hz}$.

We use two sets of five electrodes each. Electrodes in each set are tied to a velcro strap using elastic bands; two electrodes lie on the dorsal side and three on the ventral side of the forearm (see Figure 1, right panel). The first set is placed on the subject's forearm, just below the elbow; the second, on the forearm again, midway between the elbow and the wrist. This placement is intentionally largely irrespective of the (internal) anatomy of the human forearm: namely, no search for relevant muscles is performed before the straps are secured. On the other hand, rough uniform spacing around the forearm, and the use of two sets of five, are supposed to give a fair global picture of the muscular activity of the forearm. (Notice that uniform placement, irrespective of anatomy, has already been demonstrated effective, even on amputees [6].)

In order to have an indication of when the grasp was enforced, an Interlink Standard 400 FSR force-sensing resistor (see www.interlinkelectronics.com) is used. The standard amplification circuit connected to the FSR returns a voltage signal which is univocally (logarithmically) related to the force applied to its surface. The above DAQ card is used to digitise this signal, too.

\section{B. Experimental protocol}

The human subjects involved in the experiment would sit comfortably in front of a desk; then their right hand and forearm would be fitted with the electrode sets and the glove. The pressure sensor was given to the subjects to be held with the left hand. The subjects would then be instructed to initially lie their right arm relaxedly on the chair's arm, in a position such that ventral side of the right forearm would be parallel to the sagittal plane, as if to grasp cylindrically an object placed vertically (see Figure 3 to get an idea).

Under the strict request to never pronate and/or supinate the right forearm then, the subjects would be instructed to perform a reach / grasp / carry / drop / rest sequence of an object placed onto the desk lying in such a position that it would be comfortably grasped without pronating and/or supinating the forearm. Two spots would be indicated on the desk, and the subject would indifferently carry the object from one spot to the other and vice-versa. The requirement to avoid the pronation/supination movement is due to the necessity of keeping as much as possible constant the position of the electrodes with respect to the muscles of the forearm. It is easy to ascertain by palpation, actually, that the forearm skin moves dramatically with respect to the muscles, when pronating/supinating. Such an uncontrolled movement would have probably introduced too much noise in the gathered data.

The subjects were also required to press the pressure sensor with their left hand accordingly to the force employed for the grasp with the right hand.

This sequence was repeated for 20 times for each object; 5 objects were in turn used, each one to be grasped in a different way. Since 6 subjects joined in the experiment, it was expected that at the end some 600 sequences would be gathered.

The objects and grasps selected are: a DVD (flat grasp), a pen (pinch grip), a small plastic container (tripodal grip), a dry wipe marker (small power grasp) and a mug (large power grasp). Figure 2 depicts the objects and the ways to grasp them.

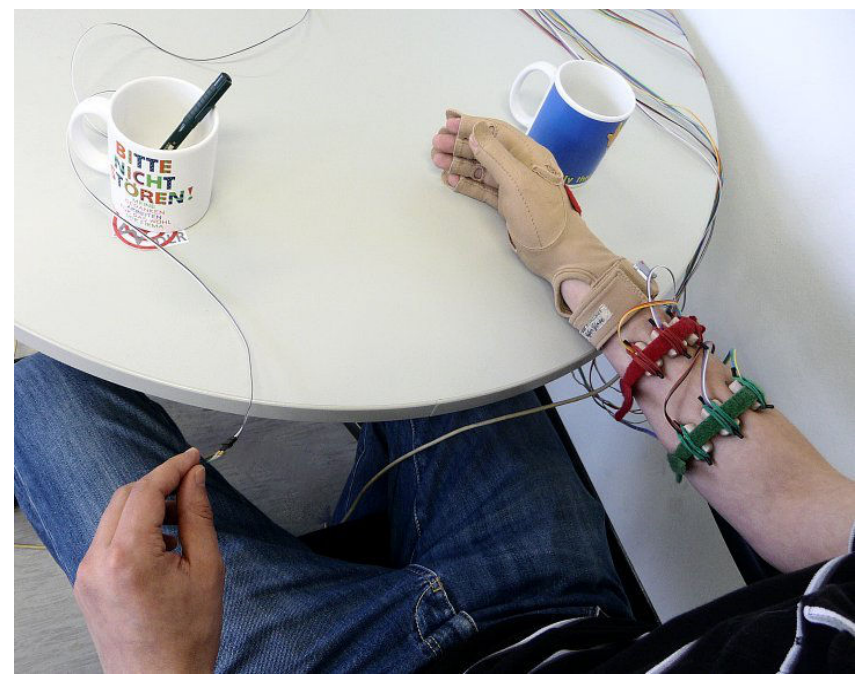

Fig. 3. Bird's eye view of the experimental setup. 

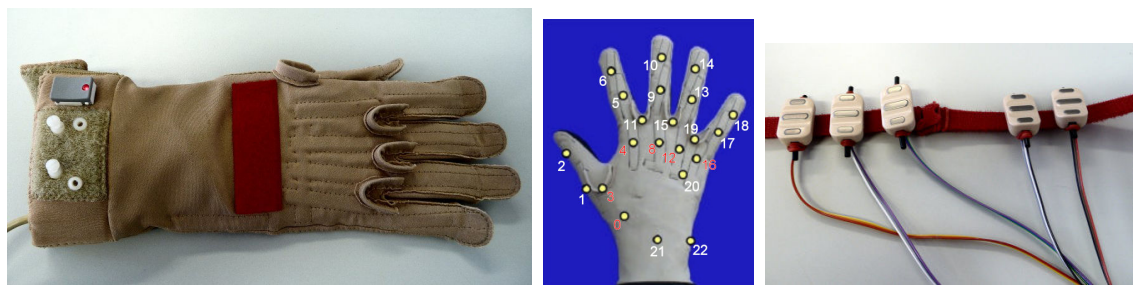

Fig. 1. Data capturing devices: (left to right) the Cyberglove; the location of its sensors (22-sensors version); 5 EMG electrodes arranged with rubber bands on a velcro strap.
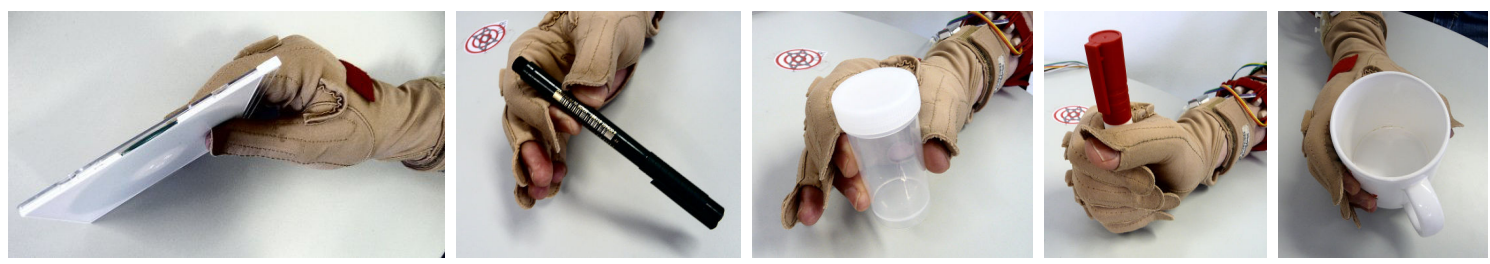

Fig. 2. The five objects, as grasped by a subject: (left to right) flat grasp, pinch grip, tripodal grip, small power grasp and large power grasp.

\section{Data synchronisation and preprocessing}

Data synchronisation is enforced on a Windows PC equipped with a multi-core processor, by gathering data from each device asynchronously and accurately timestamping each received datum. Timestamping is enforced by the HRT library [7], giving a precision of up to $1.9 \mu \mathrm{s}$. Sample-and-hold interpolation is used to find synchronised values for the electrodes, pressure sensor and glove sensors. All data are collected in batches, each one labelled with a corresponding subject and grasp index.

Initial visual inspection of the data revealed that the pressure sensor values did not correspond precisely enough to the lifting periods; therefore, a manual procedure was enforced offline, by which, for each subject and grasp sequence, the experimenter would be presented with the value of the index finger and the sum of the squares of the EMG electrodes; the experimenter would then visually identify and note the intervals corresponding to the grasps, that is the times during which the subjects were lifting the objects from one spot to the other.

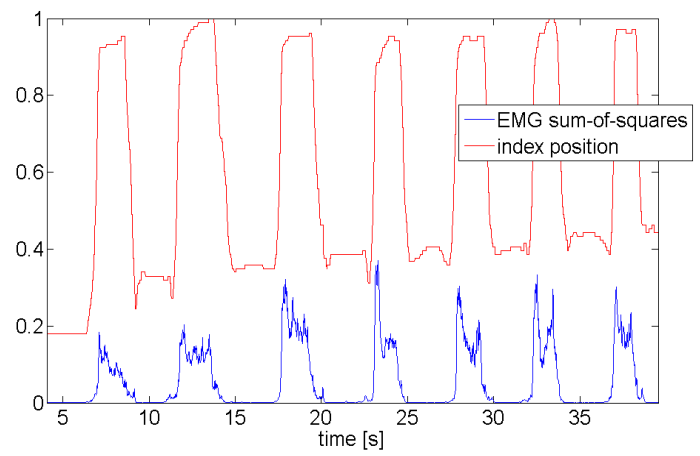

Fig. 4. Typical "good" grasping sequence (subject 1, flat grasp); correlation is apparent between the EMG power and the index finger position.

An example sequence (subject 1 doing a flat grasp) is shown in Figure 4. Correlation is apparent between the two signals; the high-valued periods denote the carrying phase, when muscle activity was maximum and the index finger would be flexed over the object, as opposed to the resting periods, characterised by low muscle activity and the index standing in the resting position (low values). It must be noted that in other cases the correlation is not apparent and that is why this process had to be manually enforced. For instance, in the large-power grasp case, the index finger would assume a lower value during lifting than in the resting phase.

Moreover, some of the lifting periods were not characterised by enough muscle activity or by the expected kinematic postures, due to local failure of the sensors. The experimenter excluded these sequences from the analysis. As well, subject 4 exhibited little or no measured muscle activity, so these data were removed from the analysis, too.

Using this procedure, $97.4 \%$ of the original data, that is 487 lifting intervals out of the expected 500 , were identified: 5 subjects repeating each of the 5 grasps for 20 times. Data were then normalised by subtracting the mean values and dividing by the standard deviations, dimension-wise. This normalisation was done per-subject, in order to remove the intra-subject differences due to the hand size and the level of muscle fitness.

All in all then, two sets of features were obtained: the 18dimensional kinematic features obtained from the glove, and the 10-dimensional $E M G$ features obtained from the EMG electrodes. Occasionally, the 5-dimensional EMG features for the forearm and elbow were considered separately. Two sets of labels were obtained, one according to the subjects $(1,2,3$, $5,6)$ and one according to the grasps, $(1,2,3,4,5$ denoting in turn the flat grasp, pinch grip, tripodal grip, small power grasp and large power grasp).

\section{EXPERIMENTAL RESULTS}

\section{A. Synergies}

Principal Component Analysis (PCA), a very basic dimensionality reduction technique (see, e.g., [8]), was first applied 

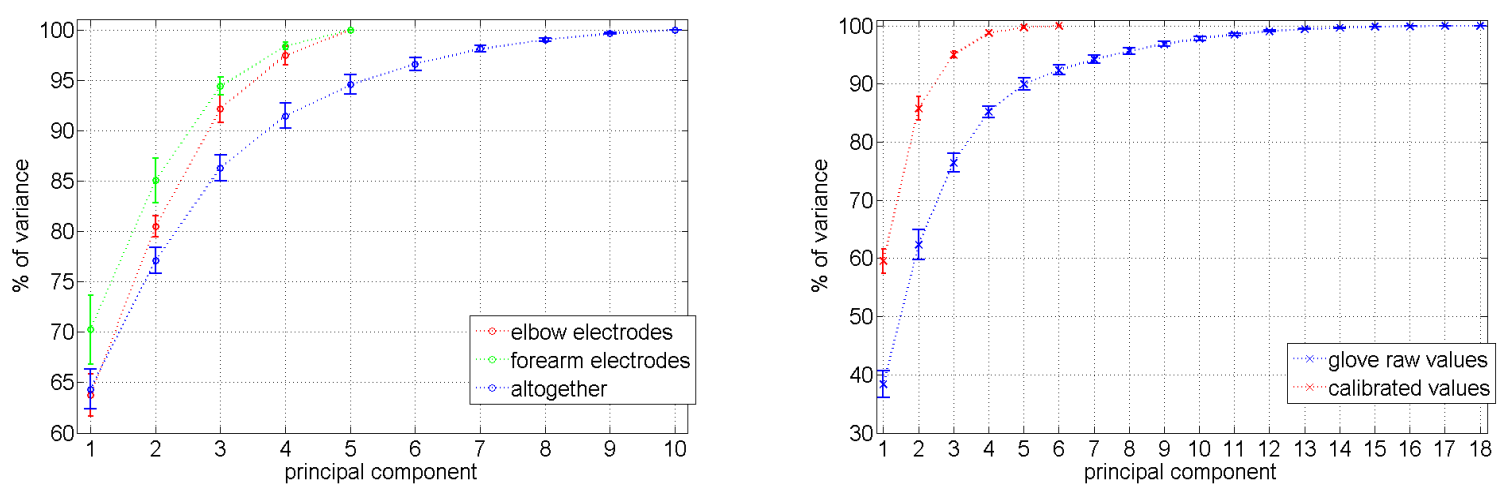

Fig. 5. Principal Component Analysis of EMG features (left) and kinematic features (right). The plots show the normalised cumulative sum of the PCA eigenvalues; markers denote the average percentage values over all subjects, and error bars are plus/minus one standard error of the mean.

to the dataset in order to check that a small number of linear combinations of kinematic and/or EMG features would account for a reasonable amount of variability in the data set.

Figure 5 shows the results. For each single subject all his grasps are considered, and PCA is applied to the resulting data subset, split according to the different features considered. The Figure shows the percentage of data variance as more principal components are considered, as a cumulative normalised sum; markers denote the average percentage values over all subjects, and error bars are placed at plus/minus one standard error of the mean. The principal components represent linear combinations of features, which in turn represent the kinematic or dynamic (EMG) activity of the subjects; therefore we will denote them, from now on, as (kinematic,dynamic) synergies as is customary. Therefore, for example, 5 EMG synergies give almost exactly $95 \%$ of the signal variance.

As is apparent from the Figure, 3 EMG synergies account for $90 \%$ to $95 \%$ of the signal variance in the forearm and elbow electrodes independently considered; and for about $86 \%$ when all electrodes are taken into account. Given the simplicity of the tasks at hand, that is, grasping in five different, very standard ways, this is in line with results found in literature (see, e.g., [3]). In the kinematic case, 4 synergies are needed when all 18 glove sensors are considered in order to reach $85 \%$ signal variance, whereas 2 of them suffice when the six calibrated values are used. This indicates that restricting the attention to the six values is actually losing much information.

This results overall means that, for all subjects independently considered, 3 EMG synergies account for most of the muscle activity; in other words, most of the grasps considered can be dynamically described using 3 numbers. This holds for each subject.

\section{B. Common synergy features}

We now consider a subset of three grasps (pinch, small power grasp and large power grasp) as performed by all subjects. The choice of these grasps stems from a very initial analysis which revealed that they are very different from each other from both a kinematic and a dynamic point of view. This is quite intuitive, and is also reflected in their distance in standard grasp taxonomies (examples can be found in [9], [10]). In this case too, data were normalised per-subject and then PCA was applied in order to be able to consider 3 EMG synergies; for visualisation purposes, we will also consider 3 kinematic synergies, although they account only for $77 \%$ of the variance.

Consider first Figure 6, depicting the grasps in 3 dimensions (first, second and third synergy) in the kinematic (left) and EMG (right) spaces. It is apparent that the grasps are well clustered, to the point that a linear classifier (i.e., a plane in 3D) could separate them perfectly from one another in kinematic space, and almost perfectly in EMG space. As opposed to this, consider now Figure 7 which depicts the same data, but associating a colour to each subject (rather than to each grasp): separability of subjects is much less clear in this case, each of them participating in each of the 3 clusters associated with the grasps. In other words, from visual inspection of the Figures, it seems that grasps are quite similar to each other in both spaces, whereas it is hard to tell one subject from another.

In order to numerically check this impression, for each of the above described settings we ran a multi-class linear classifier and considered the balanced error rate (BER) as a measure of separability. Linear classification is a statistical technique which can be used, at a very basic level such as this, to check how separated $N$ classes of objects are (see, e.g., the classic [8] again); in particular, a linear classifier will here find a set of $3 \mathrm{D}$ planes such that all samples belonging to a category will be on one side of the plane, whereas sample belonging to all other categories will be on the other side.

As a linear classifier we chose to use a Support Vector Machine (SVM) with linear kernel. SVMs [11], [12] are a machine learning method which will find the separating (hyper)plane between two sets of labelled sample, such that the margin between the categories is maximised. By margin here it is meant, twice the distance between the separating plane and the closest sample in either category. The plane thus found enjoys maximum robustness against noise in the sampling procedure [13]; in this sense, it is the optimal separating plane.

Since the dataset consists of a relatively small number of 

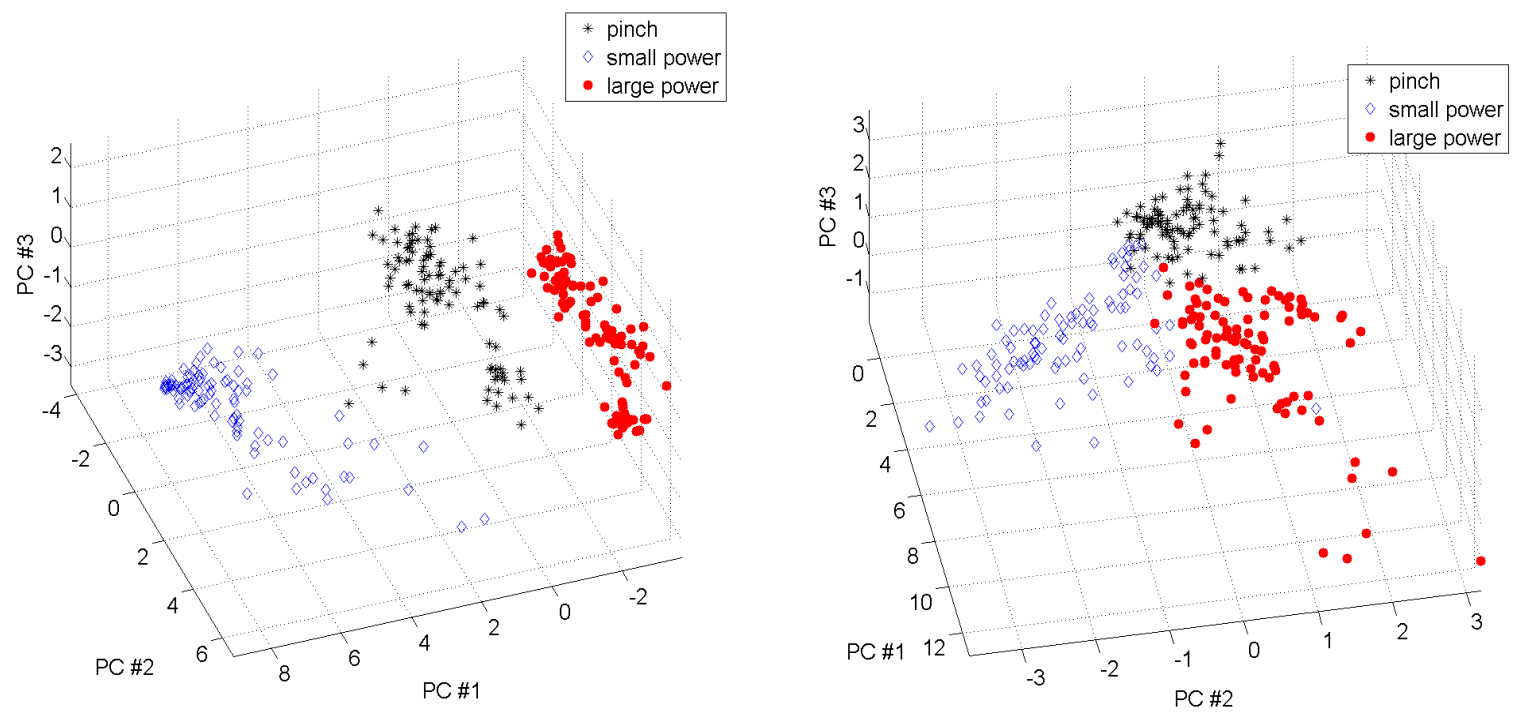

Fig. 6. 3D visualisation of 3 of the grasps as performed by all subjects; colours denote grasps. (left) Kinematic synergies, (right) EMG synergies.
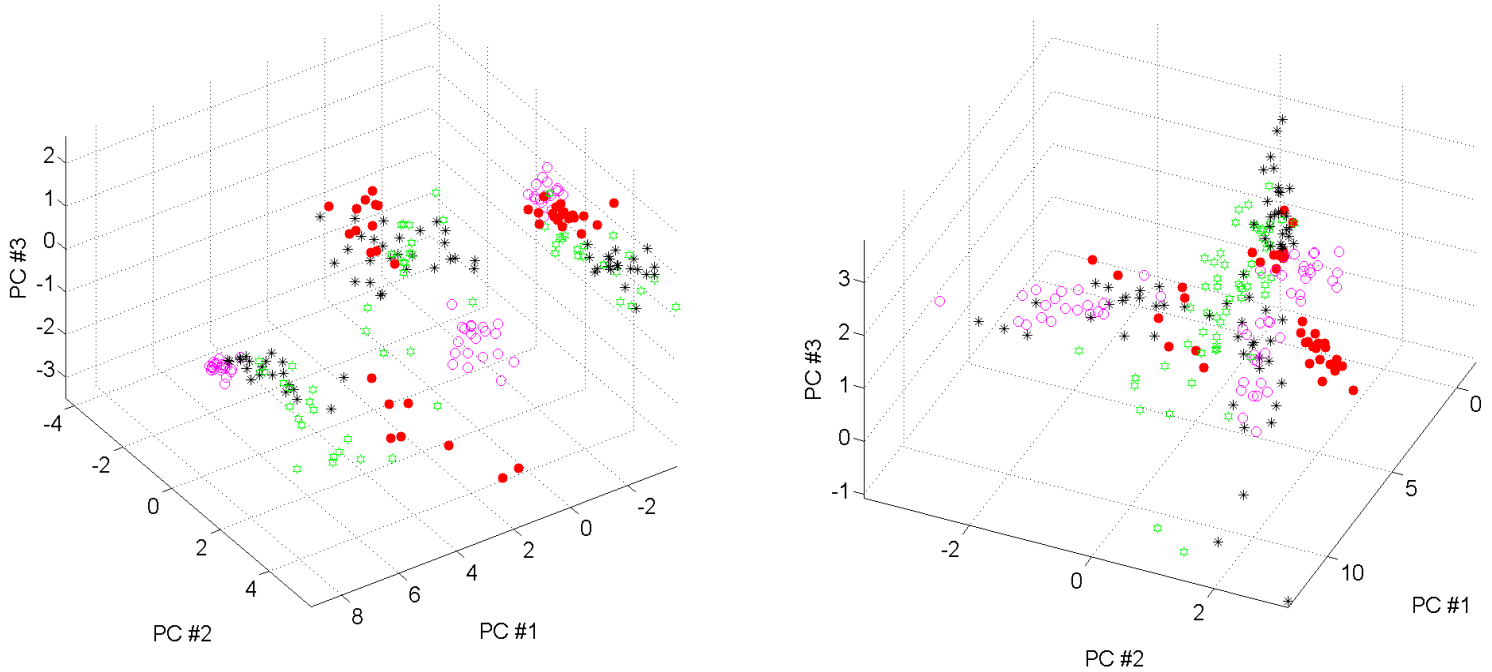

Fig. 7. 3D visualisation of 3 of the grasps as performed by all subjects; colours denote subjects. (left) Kinematic synergies, (right) EMG synergies.

samples (487), we employed 2-fold cross-validation and grid search to find the optimal SVM $C$ parameter, and then ran the procedure for 50 times, averaging then out the means and standard deviations of the errors so obtained. Table I shows the results.

As is clear from the Table, trying to separate subjects from one another is pointless, as all error rates approach chance level ( $80 \%$, since there are 5 subjects). As opposed to that, discerning grasps is accomplished quite well. In particular, kinematic synergies in this case can be perfectly discriminated (see Figure 6 (left) again-there is no error in that case) or with a high accuracy in the other cases. Particularly, using the 10 EMG electrodes altogether we achieve an error rate of

\section{$4.70 \% \pm 1.39 \%$.}

The same trend is visible when considering Table II, in which all grasps are considered. Results here are uniformly worse, as one would expect, since the flat grasp, pinch grip and tripodal grip are quite similar to each other, both from a kinematic and dynamic point of view. Still, adding this information does not help when trying to distinguish subjects from one another.

Notice, anyway, that correct classification of EMG patterns is out of the scope of this paper - that has already been done with greater success, e.g., using SVMs with Gaussian kernels. The interest of this result lies in the statistically significant separability of one or more set(s) of samples, and in this case an error rate below chance level is already meaningful. Linear 
TABLE I

BALANCED ERROR RATES OBTAINED WHILE APPLYING A LINEAR CLASSIFIER TO THE DATASETS AND LABELS SHOWN IN FIGURES 6 AND 7. CONSIDERING THREE GRASPS: PINCH GRIP, SMALL POWER GRASP AND LARGE POWER GRASP.

\begin{tabular}{l||r|r|r|r|} 
& kinematic & EMG, elbow & EMG, forearm & EMG, all \\
\hline \hline grasps & no errors & $11.62 \% \pm 2.42 \%$ & $17.19 \% \pm 2.56 \%$ & $4.70 \% \pm 1.39 \%$ \\
\hline subjects & $76.37 \% \pm 5.20 \%$ & $57.72 \% \pm 4.76 \%$ & $74.82 \% \pm 4.51 \%$ & $69.25 \% \pm 5.40 \%$ \\
\hline
\end{tabular}

TABLE II

BALANCED ERROR RATES OBTAINED WHILE APPLYING A LINEAR CLASSIFIER TO THE DATASETS AND LABELS SHOWN IN FIGURES 6 AND 7. CONSIDERING ALL GRASPS.

\begin{tabular}{l||r|r|r|r|} 
& kinematic & EMG, elbow & EMG, forearm & EMG, all \\
\hline \hline grasps & $17.88 \% \pm 2.01 \%$ & $35.56 \% \pm 3.05 \%$ & $45.58 \% \pm 2.91 \%$ & $36.08 \% \pm 2.30 \%$ \\
\hline subjects & $79.85 \% \pm 2.59 \%$ & $58.93 \% \pm 3.60 \%$ & $70.85 \% \pm 3.23 \%$ & $64.13 \% \pm 4.08 \%$ \\
\hline
\end{tabular}

separability here points at the common pattern underlying a certain class; for instance, the fact that a plane can separate the large power grasp from the pinch grip irrespective of the subjects means that an easy procedure can be found to tell which grasp is being enacted (for instance, evaluating the sample distance from the plane itself).

\section{CONCLUSIONS AND DISCUSSION}

This experiment mainly shows that EMG data gathered from 5 human subjects during a grasping task can be easily clustered on a per-grasping basis, but not on a per-subject basis. Per-subject data normalisation is carefully conthrived in order to only remove size and activation level differences among subjects, without compromising the (possible) intrasubject differences in the muscle activation patterns. By "easily clustered" here, we mean that a simple linear classifier is able to discriminate grasps, but not subjects. From this we conclude that as few as 3 dynamic synergies are sufficient to characterise one among 5 grasps, with a precision which obviously depends on the (muscular) similarity among grasps, but that anyway largely surpasses the corresponding precision when trying to discriminate humans. In one word, dynamic synergies are characterstic of grasp types and are robust across subjects.

The concept of synergies has long been established in the kinematic description of the human hand. Indeed, when taking a large number of every-day grasps into account, the movement of the fingers of the hand can be described with a very limited number of principle components (i.e., 3-5 [3], considerably less than the number of degrees of freedom of all fingers combined, i.e., 24 [14]). The novelty of our result lies in the fact that it is not clear that these synergies are also present at the level of exerted grasp force and, consequently, the EMG signal of the forearm muscles that activate the fingers. Our experiment seems to confirm that this is the case.

Potential applications of this result are mainly in force- and impedance-based control of dexterous mechanical hands; in such a case, the control system cannot probably proficiently control all the degrees of freedom of the robotic artifact, and dynamic synergies could be used to simplify the task. This would proficiently apply, as well, to hand prosthetics, especially since muscular activation level are one of the most used control methods of hand prostheses by amputees. The method can in principle be extended to any muscle complex involved in human movement, walking, manipulation.

\section{ACKNOWLEDGEMENTS}

This work is partially supported by the European FP7Project THE Hand Embodied (FP7-IST-248257) and by Swiss National Science Foundation Sinergia project Ninapro (NonInvasive Adaptive Prosthetics).

\section{REFERENCES}

[1] N. Bernstein, The coordination and regulation of movements. Oxford: Pergamon Press, 1967.

[2] F. A. Mussa-Ivaldi, S. F. Giszter, and E. Bizzi, "Linear combinations of primitives in vertebrate motor control," Proceedings of the National Academy of Sciences of the United States of America, no. 91, pp. 75347538, 1994.

[3] M. Santello, M. Flanders, and J. F. Soechting, "Postural synergies for tool use," Neuroscience, vol. 17, pp. $10105-10115,1998$.

[4] _ - "Patterns of hand motion during grasping and the influence of sensory guidance," Neuroscience, vol. 22, no. 4, pp. 1426-1435, 2002.

[5] C. Castellini and P. van der Smagt, "Surface emg in advanced hand prosthetics," Biological Cybernetics, vol. 100, no. 1, pp. 35-47, 2009.

[6] C. Castellini, E. Gruppioni, A. Davalli, and G. Sandini, "Fine detection of grasp force and posture by amputees via surface electromyography," Journal of Physiology (Paris), vol. 103, no. 3-5, pp. 255-262, 2009.

[7] J. Nilsson, "Implementing a continuously updating, high-resolution time provider for windows," The MSDN Magazine, 2004. [Online]. Available: http://msdn.microsoft.com/en-us/magazine/cc163996.aspx

[8] R. O. Duda, P. E. Hart, and D. G. Stork, Pattern Classification, 2nd ed. Wiley, 2001.

[9] M. Cutkosky, "On grasp choice, grasp models, and the design of hands for manufacturing tasks," Robotics and Automation, IEEE Transactions on, vol. 5, no. 3, pp. $269-279$, jun. 1989.

[10] S. B. Kang and K. Ikeuchi, "A grasp abstraction hierarchy for recognition of grasping tasks from observation," Proc. IEEE/RSJ Int'l Conf. on Intelligent Robots and Systems, 1993.

[11] B. E. Boser, I. M. Guyon, and V. N. Vapnik, "A training algorithm for optimal margin classifiers," in Proceedings of the 5th Annual ACM Workshop on Computational Learning Theory, D. Haussler, Ed. ACM press, 1992, pp. 144-152.

[12] V. N. Vapnik, Statistical Learning Theory. New York: John Wiley and Sons, 1998.

[13] C. J. C. Burges, "A tutorial on support vector machines for pattern recognition," Knowledge Discovery and Data Mining, vol. 2, no. 2, 1998.

[14] G. Stillfried and P. van der Smagt, "Movement model of a human hand based on magnetic resonance imaging (MRI)," in International Conference on Applied Bionics and Biomechanics (ICABB), 2010. 